\title{
Syntax production in bilinguals
}

\author{
Narly Golestani ${ }^{a, b}, *$, F.-Xavier Alario ${ }^{c}$, Sébastien Meriaux ${ }^{b}$, Denis Le Bihan ${ }^{d}$, \\ Stanislas Dehaene ${ }^{\mathrm{b}}$, Christophe Pallier ${ }^{\mathrm{b}}$ \\ a Institute of Cognitive Neuroscience, University College London, 17 Queen Square, London WC1N 3AR, UK \\ ${ }^{\mathrm{b}}$ Unité Inserm 562, Service Hospitalier Frédéric Joliot, CEA/DRM/DSV, 4 Place du Général Leclerc, 91401 Orsay Cedex, France \\ ${ }^{\mathrm{c}}$ Laboratoire de Psychologie Cognitive, CNRS \& Université de Provence, 3 Place Victor Hugo (Case 66), 13331 Marseille Cedex 3, France \\ d IFR49, UNAF, Service Hospitalier Frédéric Joliot, CEA, 4 Place du Général Leclerc, Orsay, France \\ Received 10 June 2005; received in revised form 25 September 2005; accepted 8 November 2005 \\ Available online 19 January 2006
}

\begin{abstract}
We used fMRI to examine the functional correlates of syntactical processing in the first (L1) and second (L2) languages of non-proficient, late bilinguals. Subjects either covertly read words or produced sentences from them. Syntactical production during sentence production activated regions including left inferior frontal (LIFG) gyrus and the supplementary motor area in both languages. Analyses performed on the LIFG activation identified on a subject-by-subject basis revealed greater activation in L2 compared to L1 during sentence production and during word reading, consistent with previous work suggesting that greater cognitive effort may be subserved by less well-tuned neural representations that require greater neuronal activity. Remarkably, there was a greater separation in the LIFG activations in L1 versus L2 in less compared to more proficient bilinguals during syntax production, suggesting a functional reorganisation of regions involved in syntactical production as a function of syntactical proficiency. (C) 2005 Elsevier Ltd. All rights reserved.
\end{abstract}

Keywords: Language production; Brain; fMRI; Bilingualism; Grammar

\section{Introduction}

The goal of the present study is to examine the functional neural correlates of syntactical production in the first and in the second languages of non-fluent, late bilinguals. Results of several functional neuroimaging studies on bilingualism using word generation tasks suggest shared neural circuitry for the first (L1) and second languages (L2) in proficient bilinguals having learned their second language after the age of 5 (Klein, Zatorre, Milner, Meyer, \& Evans, 1994; Vingerhoets et al., 2003). Other studies using sentence perception tasks have also shown activation of overlapping brain regions for both languages in both late and early high proficiency bilinguals (Perani et al., 1998). Two fMRI studies, however, have shown different results. Dehaene et al. (1997) showed that language comprehension during passive listening to speech activates similar regions in L1, but that areas activated by L2 are more variable in moderately fluent bilinguals having learned their second language after the age

\footnotetext{
* Corresponding author. Tel.: +44 207679 7529; fax: +44 2079168517.

E-mail address: narlyg@bic.mni.mcgill.ca (N. Golestani).
}

of 7. Kim, Relkin, Lee, and Hirsch (1997) showed that during covert production of sentences, frontal activations overlapped in early bilinguals but were in separate regions in late bilinguals. In this latter study, it appears that proficiency may have been confounded with age of acquisition.

The findings from the two latter studies suggest that when the second language is not completely mastered or when it is learned late in life, regions subserving the perception and production of this second language differ from those subserving these processes in the first, native language. These results do not, however, allow to determine which level of language such differences in the functional architecture of L1 and L2 arise from. It is possible, for example, that such differences result from syntactic but not from phonetic nor from semantic processing (Wartenburger et al., 2003). Indirect support for this idea comes from electrophysiological (Weber-Fox \& Neville, 1996) and behavioural (Birdsong, 1999) evidence that the use of grammar is much more adversely affected by later ages of exposure than is the use of lexical items, and that the neural systems mediating grammatical processing are more vulnerable to changes in early experience than are those mediating semantic processing. There is also evidence that in adults, the pattern of neural activation during the 
perception of newly acquired phonetic contrasts is similar to that arising from the perception of native speech sounds, suggesting that neural systems mediating phonetic processing are not different, whether new speech sounds are learned early or later in life (Golestani \& Zatorre, 2004). More generally, the finding that semantic versus phonological and syntactic processing recruit different subregions of the left inferior frontal lobe (Caplan, Alpert, \& Waters, 1998; Caplan, Alpert, Waters, \& Olivieri, 2000; Dapretto \& Bookheimer, 1999; Wagner, PareBlagoev, Clark, \& Poldrack, 2001), suggests that separate neural systems subserve different levels of language.

Only one functional imaging study, to our knowledge, has investigated language production in L1 and L2 in bilinguals at the sentence level (Kim et al., 1997). The task employed however, does not allow to determine whether or not subjects were actually performing the task at hand, nor does it offer control over the cognitive processing during the covert production task. For example, different subjects could have been producing utterances involving different levels of grammatical and/or semantic complexity or difficulty in L1 versus L2. Last, as noted above, it does not allow one to disentangle different levels of language. We propose to use fMRI to examine the functional correlates of syntactical processing in the first and second languages of moderately fluent bilingual subjects, during a covert production task. In addition, we will test for relationships between the functional imaging results and grammatical proficiency across subjects.

\subsection{Previous functional imaging work on syntax}

Most neuroimaging studies specifically examining syntactic processing, or contrasting it with semantic processing, have used receptive rather than production tasks. Many of these studies have found activation in Broca's area (BA 44/45) (Embick, Marantz, Miyashita, O'Neil, \& Sakai, 2000; Dapretto \& Bookheimer, 1999; Embick et al., 2000). Only one study, to our knowledge, has specifically examined the neural correlates of syntax in bilinguals. Wartenburger et al. (2003) used fMRI to investigate the effects of age of acquisition (AOA) and proficiency level (PL) on the neural correlates of grammatical and semantic judgements during syntactic and semantic anomaly detection tasks. They found that while the pattern of brain activity for semantic judgment was largely dependent on PL, AOA mainly affected the cortical representation of grammatical processes. Specifically, during grammaticality judgements, they found that late proficient bilinguals activate inferior frontal regions bilaterally more than do early proficient bilinguals.

Very few studies have used production tasks to specifically examine syntax. Heim, Opitz, and Friederici (2002) found activation of a superior portion of Broca's area during a gender selection task. Indefrey et al. (2001) did a PET study using a restrictive scene description paradigm in which subjects produced utterances to describe a simple geometrical animated scene in three different prespecified ways: with full sentences, with a sequence of noun phrases that had local syntactic structure, or with single words having no syntactic relationship. They found that the left rolandic operculum (BA 6), caudally adjacent to Broca's area, is involved in both sentence-level and local (phrase-level) syntactic encoding. This finding was replicated using fMRI (Indefrey, Hellwig, Herzog, Seitz, \& Hagoort, 2004).

Results of other studies suggest that syntax does not involve one specific brain region but that it involves a network of areas including Broca's area, sometimes extending to BA 46, 47, 6 , and 9 , the anterior temporal lobe including the temporal pole (BA38), often bilaterally, the left or bilateral superior and middle temporal gyri (BA 21/22), the left angular/supramarginal gyri (BA 39/40), the cingulate gyrus, and finally the basal ganglia (Dapretto \& Bookheimer, 1999; Keller, Carpenter, \& Just, 2001). Due to different results obtained across studies using different stimuli and tasks, it has been argued that these areas are not uniquely involved in syntax, but that different parts of the network are recruited for different aspects of syntactic processing. For example, the middle and superior temporal lobes might be involved in the lexical and phonological processing associated with the stimuli, the anterior temporal lobe may be involved in combining the activated information or encoding the information for later use, and Broca's area might be involved in storing material when processing load increases (Kaan \& Swaab, 2002). More generally, Broca's area is also thought to be involved in other aspects of language such as phonology, suggesting that it plays multiple roles in language. Further, it is thought to be involved in non-linguistic processing such as non-verbal learning and working memory (Klingberg, Kawashima, \& Roland, 1996), imitation of sequences of motor gestures (Rizzolatti \& Arbib, 1998), and representing and processing hierarchical structure (Friederici, 2004; Tettamanti et al., 2002). These functions are all likely components of syntax processing, and some have therefore argued that Broca's area performs functions that are necessary but not specific to syntax. For example, it has been proposed that the role of the inferior frontal cortex in working memory makes it indispensable during syntactic decoding and sentence generation (Müller \& Basho, 2004). The exact role of Broca's area during grammar processing, however, is a topic of heated debate, and it is beyond the scope of this article to address these issues.

In the current study, we used fMRI to examine the functional correlates of syntactical production in the first and second language of 12 moderately fluent, late bilinguals. During scanning, subjects viewed series of either three or five words either in L1 or in L2, and were required to either covertly read the words (baseline condition), or to covertly produce a simple grammatical sentence from the words (experimental condition). We addressed the following questions. First, does syntactical production recruit similar or different regions of the brain in the first versus the second language of bilingual individuals? Second, is the extent or strength of activation different in the two languages? Third, does grammatical proficiency in L2, as measured by a TOEFL subtest (see Section 2) modulate activity observed during the syntactical production task in L2? We predicted firstly that in both languages, the word reading condition would activate regions previously shown to be activated during covert reading, including occipital and occipito-temporal regions, primary motor regions, the left middle and/or inferior temporal cortices, and possibly Broca's area (Bookheimer, Zeffiro, Blaxton, Gaillard, \& Theodore, 1995; Joubert et al., 
2004). Overlap in brain activity in L1 and L2 was predicted based on results of neuroimaging studies of language function in healthy bilinguals, some of which show that at the single word level, brain regions subserving the native (L1) and second language (L2) appear to overlap (Chee et al., 1999; Illes et al., 1999; Klein et al., 1994). We also predicted that in both L1 and L2, the sentence minus word subtraction would activate regions previously implicated in syntactical processing, including Broca's area (BA 44/45) and possibly adjacent left prefrontal regions including Brodman's areas 6, 9, 46, and 47, the basal ganglia, left inferior parietal lobe, and maybe temporal regions. Further, based on previous work showing that frontal regions appear to be more active during lesser language proficiency (Chee, Hon, Lee, \& Soon, 2001) or poorer task performance (Golestani \& Zatorre, 2004), we predicted that the left prefrontal cortex would be more active in L2 compared to L1 during sentence production and during word reading, and maybe also during the production of syntax. Lastly, based on above mentioned work showing nonoverlapping frontal activations in late compared to early bilinguals (Kim et al., 1997), we were interested in testing for similar effects with respect to L2 proficiency, using more controlled and targeted syntax and sentence generation tasks. Specifically, we predicted greater separation in left prefrontal activations for L1 versus L2 in less compared to more proficient bilinguals.

\section{Methods}

\subsection{Participants}

Participants were 12 right-handed native French speakers (seven men), ranging in age from 20 to 28, who started to learn English at the ages of 10-12 and who studied it for a total of 5-7 years in school. None had ever lived in an English-speaking environment. All were moderately fluent bilinguals, however, the level of L2 proficiency varied across individuals. Grammatical proficiency was assessed with the Structure subtest of the Toefl test of English as a Foreign Language (TOEFL). This subtest specifically evaluates the grammatical level, and includes sentence completion and error identification items. The test includes up to 16 different grammar points, ranging from adjectives to subordination (see http://www.free-toefl.com/Tools/Tests.aspx). All subjects gave informed consent to participate in the study, which was approved by the regional ethical committee.

\subsection{Experimental and control tasks}

Subjects viewed either three or five words either in French (L1) or in English (L2) presented all at once on the screen, and were required to either covertly read the words (baseline condition), or produce a simple sentence from the words (experimental condition). The two task conditions, two word length conditions, and two language conditions yielded a total of eight conditions. The conditions occurred in miniblocks, pseudorandomised in order, each lasting $30 \mathrm{~s}$, starting with a visually presented instruction for each condition ('Français Mots', 'Français Phrases', 'English Words', 'English Sentences') lasting $2 \mathrm{~s}$, and followed by five trials lasting $5.6 \mathrm{~s}$ each. A silent period of $8 \mathrm{~s}$ separated each miniblock and preceded the first one. Each session contained two mini-blocks per condition plus silent periods, and lasted approximately $10 \mathrm{~min}$. Each subject performed three sessions during scanning, yielding a total of six mini-blocks per condition.

For the sentence condition, subjects were instructed to generate a simple sentence from the words appearing on the screen by adding determiners, prepositions, and particles, and by conjugating verbs when necessary, but without adding content words such as nouns, verbs, adjectives, or adverbs. They were told that there was no right or wrong answer, but that the sentences generated had to be grammatically correct. The word presentation order during the word reading condition was such that one would have to reorder the words in order to generate a grammatical sentence. During the sentence generation condition, however, words were presented in the correct order for generating a sentence. This was done in order to (1) ensure that the sentence generation task was not a word ordering one nor one that placed heavy demands on verbal working memory, and (2) to minimise the chances that the word presentation order 'primes' the implicit or automatic generation of a sentence. Examples of the stimuli are as follows: word reading, English: 'build workers house' or 'other do John play children', French: 'courses étudiants faire' or 'près aller mère promener océan', sentence generation, English: 'family eat meal' or 'she go with Dave concert', French: 'amis marcher plage' or 'petit garçon vouloir aider parents'.

Subjects were trained on all conditions before the scan. During the first training session, they produced the words and sentences overtly, both in order to ensure that the tasks were performed correctly, and to give feedback to the subject in case they were not. They then performed the tasks covertly (silently). In both overt and covert conditions, they were required to press a button as soon as they had finished generating the words or the sentences, as they would later be required to do during scanning. Training therefore also served to collect response times during both overt and covert production; these could later be compared to those obtained during covert production during scanning as an indirect index of task performance (Buckner, Koutstaal, Schacter, \& Rosen, 2000).

\section{3. fMRI Scanning protocol}

Imaging was performed on a 3-T whole body system (Bruker, Germany). Three series of 307 gradient-echo images of blood-oxygenation-level-dependent (BOLD) signal were acquired $(\mathrm{TR}=2 \mathrm{~s}, \mathrm{TE}=30 \mathrm{~ms}, 64 \times 64 \times 22$ images; $3.75 \mathrm{~mm} \times 3.75 \mathrm{~mm} \times 5 \mathrm{~mm}$ voxels; 22 slices). High-resolution T1 weighted anatomical scans $(1 \mathrm{~mm} \times 1 \mathrm{~mm} \times 1.2 \mathrm{~mm}$ voxels $)$ were also obtained.

\subsection{Analyses}

\subsubsection{Imaging analyses}

Data were analysed using SPM99 (Friston et al., 1995), starting with slice timing, distortion correction, spatial realignment (correction for movement), spatial normalization and smoothing with a $5 \mathrm{~mm}$ Gaussian kernel. The statistical analyses were performed by generating a linear model by defining 10 categories of events: English words 3, English words 5, English sentences 3, English sentences 5, French words 3, French words 5, French sentences 3, French sentences 5, instructions, and silence. These categories were crossed with indicator variables for the three sessions, yielding 10 onset vectors per session, which were convolved with an ideal heamodynamic response curve and its derivative (included to model small temporal shifts). Both individual and group analyses were performed. The group analyses consisted of $t$-tests using the individual contrast images smoothed at $8 \mathrm{~mm}$ (one image per subject). We also performed correlational analyses, in which we correlated the $t$-maps representing contrasts of interest with a behavioural measure (performance on the Toefl subtest). All individual and group results were examined at the voxel-wise threshold of $p<0.001$ (uncorrected) and a cluster extent threshold of $p<0.05$ (corrected).

\subsubsection{Subject-by-subject analyses}

The location of the left inferior frontal activation was very variable across subjects. We therefore performed analyses on various measures obtained from activation clusters in this region identified on a subject-by-subject basis. We first identified significant left prefrontal activation clusters for each subject, taken at a threshold of $p<0.001$, in the sentence minus word subtraction in both English and French (Kim et al., 1997). For the one subject showing right instead of left prefrontal activation in the syntactical contrast (see Results section below), we performed the below analyses on the prefrontal cluster identified in the right hemisphere. Also, for the analyses on the location of the ROIs (see below), we changed the sign of the $x$-axis coordinates of the left prefrontal cluster for this subject. We tested for differences between L1 and L2 in (a) the strength of the LIFG activation, as measured by the percent signal change in this cluster, (b) the size of the LIFG activation, as measured by voxel counts in the ROI at an activation threshold of $p<0.001$, and (c) the location of the 
LIFG activation, as measured by the $x, y$, and $z$ coordinates of the location of the peak activation. We also tested for possible relationships between a measure of syntactical proficiency, as measured by the Toefl subtest, and (a) the strength of activation (measured as above), (b) the location of activation (measured as above), and (c) the size of the activation (measured as above). Last, we tested for a relationship between Toefl scores and the distance (in millimetres) separating the location of the peak activation across subjects.

\section{Results}

Results from two subjects had to be excluded because of motion artefact that could not be eliminated with motion correction, and neither by using motion correction parameters as regressors in the data analyses. The DISTANCE SPM99/2 toolbox (Kherif et al., 2003) was used to assess the global intersubject variability in the fMRI data at the population level, before launching a random effect analysis. A similarity measure was used to compute distances between subjects and detect possible atypical data. This method confirmed the presence of the two outliers described above. From now on, only results from the remaining 10 subjects will be presented and discussed.

\subsection{Behavioural findings}

Scores on the Toefl subtest ranged from 10 to 19 out of 20 (S.D. =3.0). During overt production, all subjects accurately read words and produced sentences in all of the conditions. See Fig. 1 for response times. A four-way repeated measures analysis of variance (ANOVA) (language $\times$ task $\times$ length $\times$ modality (overt/covert)) on the response times showed that the main effect of modality is not significant $(F(9)=0.09, p>0.05)$. In order to indirectly evaluate whether or not subjects were performing the task at hand during scanning, we compared the pattern of results obtained using ANOVAs on response times obtained during covert production during scanning versus overt production during training. A three-way repeated measures ANOVA on language (French/English), number of words (3 versus 5), and task (sentences versus words) during overt production revealed a main effect of language $(F(9)=49.4, p<0.001)$ (English $>$ French), word number $(F(9)=430.9, p<0.001)(5>3)$, and of task $(F(9)=17.0$, $p<0.001$ ) (sentences $>$ words). Only the language by task interaction was significant $(F(9)=20.2, p<0.005)$. Tukey post hoc analyses revealed that producing English sentences takes longer than reading English words $(t(2,9)=10.54, p<0.01)$, and that producing French sentences takes longer than reading French words $(t(2,9)=4.20, p<0.05)$. Note that the task effect is somewhat weaker in French compared to English.

A similar pattern of results was revealed for covert response results. A three-way ANOVA revealed main effects of language $(F(9)=3.91, p=0.05)$ (English $>$ French), word number $(F(9)=366.0, p<0.001)(5>3)$, and of task $(F(9)=43.6$, $p<0.001$ ) (sentences $>$ words). The language by task interaction was marginally significant $(F(9)=4.1, p=0.07)$. Tukey post hoc analyses revealed that once again, producing English sentences takes longer than reading English words $(t(2,9)=7.66$, $p<0.01$ ), and that producing French sentences takes longer than reading French words $(t(2,9)=4.30, p<0.05)$. Once again, the task effect is somewhat weaker in French compared to English. The similar pattern of response times across modalities suggests that subjects were indeed performing the task at hand during covert production during fMRI scanning.

\subsection{Functional activation findings}

\subsubsection{Group subtractions}

Table 1 provides the stereotaxic coordinates for the main effect of words in English and French. In both languages, the word reading versus silence subtraction yielded activation in the left premotor cortex (BA 6), and in the occipital (BA 18) and fusiform (BA 19/37) regions bilaterally. In English, there was additional activation in the left putamen. Table 2 presents the results for the main effects of sentences in L1 and L2. Sentence production versus silence also yielded a similar pattern of activation across languages, in regions including the left inferior frontal gyrus (BA 44), which in English, extended to BA 45 and BA 46. Table 3 and Fig. $2 \mathrm{~A}$ and $\mathrm{B}$ present the results of the sentence minus word, 'syntactical' subtraction. Most subjects had left frontal activations for this contrast. In English, nine out of 10 subjects show activations in this region in English and one out of 10 shows activation in the right hemisphere homologue of Broca's area, and in French, seven out of 10 activate the left prefrontal and one out of 10 the right prefrontal cortex. Note, however, that the exact location of the activations varied across individuals. Table 4 shows the results for the task by language interactions, and finally, Table 5 presents the results of the word length effect (5-3) for the word reading and for the sentence generation conditions, collapsed across languages.
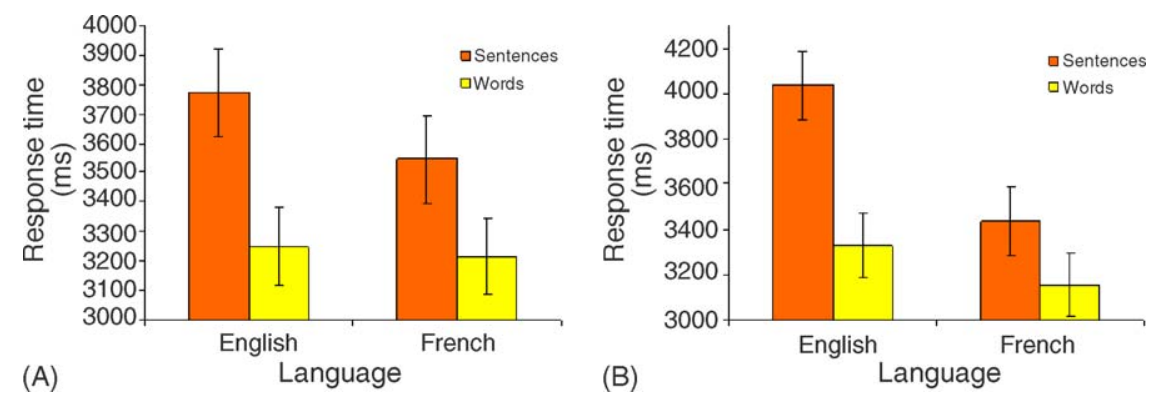

Fig. 1. Behavioral results. Response times showing language by task interaction during (A) covert and (B) overt production. Error bars represent standard error values. 
Table 1

Words vs. silence subtraction

\begin{tabular}{|c|c|c|c|c|c|c|c|c|c|c|c|}
\hline \multirow[t]{3}{*}{ Structure } & \multicolumn{5}{|c|}{ English } & \multirow[t]{3}{*}{ BA } & \multicolumn{5}{|c|}{ French } \\
\hline & \multicolumn{3}{|c|}{ Talaraich coordinates } & \multirow[t]{2}{*}{$t$-Value } & \multirow[t]{2}{*}{$k$} & & \multicolumn{3}{|c|}{ Talaraich coordinates } & \multirow[t]{2}{*}{$t$-Value } & \multirow[t]{2}{*}{$k$} \\
\hline & $x$ & $y$ & $z$ & & & & $x$ & $y$ & $z$ & & \\
\hline $\mathrm{L}$ premotor cortex & -56 & 0 & 44 & 7.38 & 87 & 6 & -48 & -16 & 56 & 7.19 & 125 \\
\hline L occipital cortex & -28 & -88 & 0 & 11.01 & 186 & 18 & -32 & -88 & 4 & 13.83 & 152 \\
\hline R occipital cortex & 32 & -84 & 4 & 8 & 99 & 18 & 32 & -84 & 4 & 8.41 & 85 \\
\hline $\mathrm{L}$ fusiform $\mathrm{g}$ & -48 & -64 & -12 & 10.31 & $(186)$ & $19 / 37$ & -48 & -64 & -12 & 5.53 & (125) \\
\hline $\mathrm{R}$ fusiform $\mathrm{g}$ & 40 & -72 & -20 & 6.59 & (99) & $19 / 37$ & 40 & -76 & -16 & 5.80 & $(85)$ \\
\hline L putamen & -24 & -8 & 4 & 9.88 & 92 & & & & & & \\
\hline
\end{tabular}

Notes: All results are presented at the voxel-wise threshold of $p<0.001$ (uncorrected) and a spatial extent threshold of $p<0.05$ (corrected). Also, cluster sizes reported in brackets refer to activations, which were within clusters listed elsewhere in the table.

Table 2

Sentences vs. silence subtraction

\begin{tabular}{|c|c|c|c|c|c|c|c|c|c|c|c|}
\hline \multirow[t]{3}{*}{ Structure } & \multicolumn{5}{|c|}{ English } & \multirow[t]{3}{*}{$\mathrm{BA}$} & \multicolumn{5}{|c|}{ French } \\
\hline & \multicolumn{3}{|c|}{ Talaraich coordinates } & \multirow[t]{2}{*}{$t$-Value } & \multirow[t]{2}{*}{$k$} & & \multicolumn{3}{|c|}{ Talaraich coordinates } & \multirow[t]{2}{*}{$t$-Value } & \multirow[t]{2}{*}{$k$} \\
\hline & $x$ & $y$ & $z$ & & & & $x$ & $y$ & $z$ & & \\
\hline L IFG & -48 & 12 & 20 & 15.14 & 287 & 44 & -52 & 8 & 20 & 5.91 & 269 \\
\hline L IFG & -36 & 24 & 8 & 6.5 & (287) & 45 & & & & & \\
\hline L MFG & -48 & 24 & 24 & 4.86 & (287) & 46 & & & & & \\
\hline L premotor cortex & -56 & -4 & 36 & 9.57 & (287) & 6 & -52 & -4 & 48 & 13.71 & (269) \\
\hline L occipital cortex & -32 & -84 & 0 & 14.80 & 247 & 18 & -28 & -88 & 0 & 9.63 & 178 \\
\hline R occipital cortex & 32 & -84 & 4 & 14.81 & 290 & 18 & 32 & -84 & 4 & 8.42 & 140 \\
\hline $\mathrm{R}$ cerebellum & 20 & -60 & -24 & 12.6 & (290) & & 36 & -72 & -20 & 5.53 & (140) \\
\hline $\mathrm{L}$ fusiform $\mathrm{g}$ & -44 & -60 & -12 & 10.04 & (247) & $19 / 37$ & -44 & -60 & -12 & 6.26 & (178) \\
\hline $\mathrm{R}$ fusiform $\mathrm{g}$ & 40 & -76 & -12 & 8.41 & (290) & $19 / 37$ & 40 & -76 & -12 & 4.91 & (140) \\
\hline L inf. parietal & -52 & -36 & 52 & 10.27 & 150 & 40 & & & & & \\
\hline
\end{tabular}

Notes: All results are presented at the voxel-wise threshold of $p<0.001$ (uncorrected) and a spatial extent threshold of $p<0.05$ (corrected). Also, cluster sizes reported in brackets refer to activations, which were within clusters listed elsewhere in the table.

\subsubsection{Correlations with Toefl}

In order to examine the relationship between brain activity and syntactical proficiency, we performed a correlational analysis between the BOLD signal during the English sentence minus word contrast and the Toefl subtest scores. There were significant positive correlations in the left occipital cortex and in the left caudate nucleus/putamen (Table 6). There were no significant negative correlations, even at the lower threshold of $p=0.01$.

Table 3

Sentences vs. words subtraction

\begin{tabular}{|c|c|c|c|c|c|c|c|c|c|c|c|}
\hline \multirow[t]{3}{*}{ Structure } & \multicolumn{5}{|c|}{ English } & \multirow[t]{3}{*}{$\mathrm{BA}$} & \multicolumn{5}{|c|}{ French } \\
\hline & \multicolumn{3}{|c|}{ Talaraich coordinates } & \multirow[t]{2}{*}{$t$-Value } & \multirow[t]{2}{*}{$k$} & & \multicolumn{3}{|c|}{ Talaraich coordinates } & \multirow[t]{2}{*}{$t$-Value } & \multirow[t]{2}{*}{$k$} \\
\hline & $x$ & $y$ & $z$ & & & & $x$ & $y$ & $z$ & & \\
\hline L IFG & -52 & 8 & 16 & 11.91 & 310 & 4 & -52 & 8 & 16 & 6.05 & 247 \\
\hline L IFG & -40 & 24 & 4 & 8.42 & $(310)$ & 5 & -44 & 28 & 4 & 5.62 & (247) \\
\hline L MFGs & -44 & 28 & 24 & 20.5 & (310) & 6 & -52 & 32 & 20 & 6.25 & (247) \\
\hline Pre-SMA & -12 & 8 & 56 & 5.91 & 49 & & -12 & 8 & 60 & 8.04 & 30 \\
\hline L sup. parietal & -36 & -52 & 48 & 7.65 & (141) & & & & & & \\
\hline R occipital cortex & 36 & -84 & 4 & 4.83 & 24 & 8 & & & & & \\
\hline $\mathrm{R}$ cerebellum & 32 & -60 & -20 & 9.22 & 18 & & & & & & \\
\hline $\mathrm{L}$ precentral $\mathrm{g}$ & & & & & & 6 & -48 & 4 & 16 & 8.87 & (247) \\
\hline L putamen & & & & & & & -20 & -4 & 12 & 8.89 & 84 \\
\hline
\end{tabular}

Notes: All results are presented at the voxel-wise threshold of $p<0.001$ (uncorrected) and a spatial extent threshold of $p<0.05$ (corrected). Also, cluster sizes reported in brackets refer to activations, which were within clusters listed elsewhere in the table. 


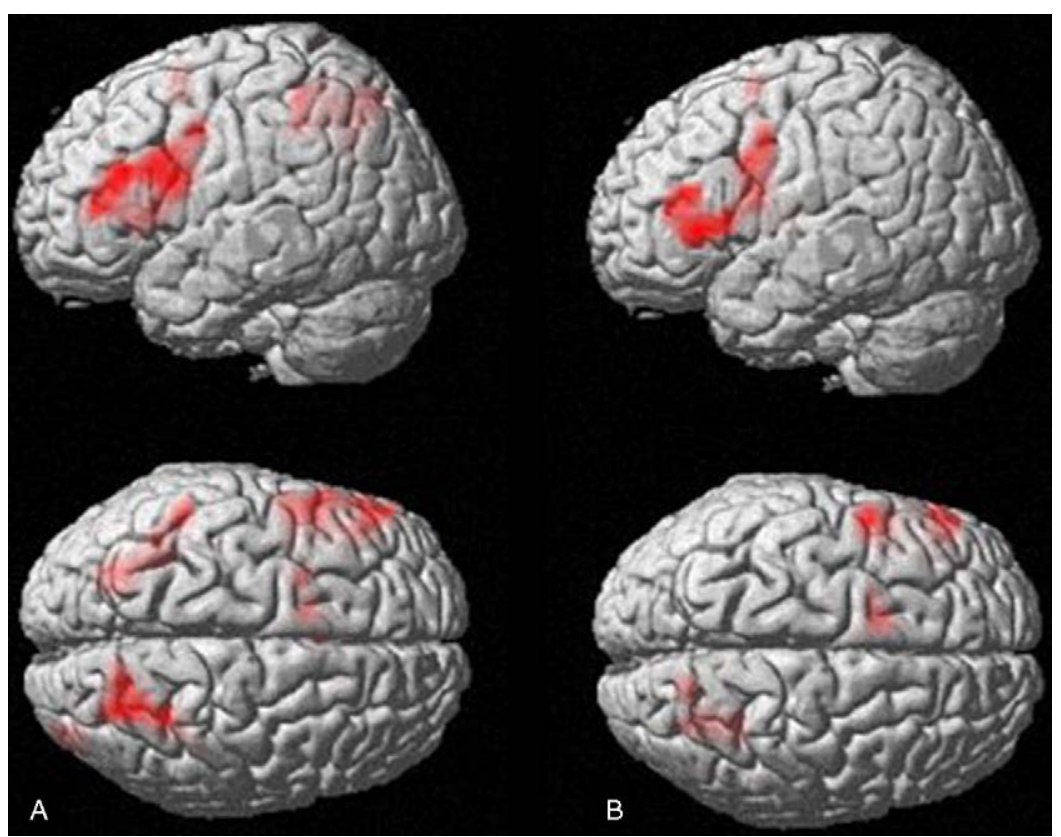

Fig. 2. Group maps: brain activity during syntactical task. Left hemisphere and top views of 3D renderings of group activations for the sentence production vs. word reading conditions in (A) English and in (B) French (thresholded at $p=0.001$ (uncorrected), with a spatial extent threshold set at $p=0.05$ (corrected)).

\subsubsection{Subject-by-subject analyses}

Based on results of previous research showing that frontal regions appear to be more active during lesser language proficiency (Chee et al., 2001) or poorer task performance (Golestani \& Zatorre, 2004), we had predicted activation in Broca's area in the task by language interaction, which, however, yielded no significant results. Individual differences in the location of the left prefrontal activation during the syntactical task may have made it difficult to detect an effect in this region in group subtractions. We therefore identified significant left prefrontal activation clusters in the sentence minus word subtraction in both English and
French on a subject-by-subject basis (see Section 2). In order to test for language and task differences in the strength of the LIFG activation, we extracted the percent signal change for each subject at the peak $t$-value location in this cluster during each of the four experimental conditions, and performed a two-way repeated-measures ANOVA (language by task) on these values. We found main effects of language $(F(7)=14.3, p<0.01)$ indicating a greater signal change in English than in French, and of task $(F(7)=54.2, p<0.001)$ indicating greater signal change during sentence production than in word reading. The interaction was not significant. Results of $t$-tests on the location of the

Table 4

Interactions: sentences vs. words

\begin{tabular}{|c|c|c|c|c|c|c|c|c|c|c|}
\hline \multirow[t]{3}{*}{ Structure } & \multicolumn{4}{|c|}{ English-French } & \multirow[t]{3}{*}{ BA } & \multicolumn{5}{|c|}{ French-English } \\
\hline & \multicolumn{3}{|c|}{ Talaraich coordinates } & \multirow[t]{2}{*}{$t$-Value } & & \multicolumn{3}{|c|}{ Talaraich coordinates } & \multirow[t]{2}{*}{$t$-Value } & \multirow[t]{2}{*}{$k$} \\
\hline & $x$ & $y$ & $z$ & & & $x$ & $y$ & $z$ & & \\
\hline
\end{tabular}

Notes: All results are presented at the voxel-wise threshold of $p<0.001$ (uncorrected) and a spatial extent threshold of $p<0.05$ (corrected). Also, cluster sizes reported in brackets refer to activations, which were within clusters listed elsewhere in the table.

Table 5

Five minus three

\begin{tabular}{|c|c|c|c|c|c|c|c|c|c|c|c|}
\hline \multirow[t]{2}{*}{ Structure } & \multicolumn{5}{|c|}{ Words } & \multirow[t]{2}{*}{ BA } & \multicolumn{5}{|c|}{ Sentences } \\
\hline & $x$ & $y$ & $z$ & $t$-Value & $k$ & & $x$ & $y$ & $z$ & $t$-Value & $k$ \\
\hline L occipital cortex & -20 & -92 & 8 & 7.45 & 309 & 18 & -24 & -92 & 16 & 11.64 & 221 \\
\hline $\mathrm{L}$ fusiform & -44 & -76 & -12 & 7.44 & (309) & $19 / 37$ & & & & & \\
\hline $\mathrm{R}$ occipital cortex & & & & & & 18 & 32 & -84 & 16 & 7.43 & 61 \\
\hline
\end{tabular}

Notes: All results are presented at the voxel-wise threshold of $p<0.001$ (uncorrected) and a spatial extent threshold of $p<0.05$ (corrected). Also, cluster sizes reported in brackets refer to activations, which were within clusters listed elsewhere in the table. 
Table 6

Correlation between English Sentences minus Words subtraction and Toefl scores

\begin{tabular}{|c|c|c|c|c|c|c|}
\hline \multirow[t]{2}{*}{ Structure } & \multicolumn{3}{|c|}{ Talaraich coordinates } & \multirow[t]{2}{*}{$t$-Value } & \multirow[t]{2}{*}{$k$} & \multirow[t]{2}{*}{$\mathrm{BA}$} \\
\hline & $x$ & $y$ & $z$ & & & \\
\hline \multicolumn{7}{|l|}{ Positive correlations } \\
\hline Left caudate nucleus/putamen & -8 & 12 & -8 & 7.89 & 35 & \\
\hline Left occipital cortex & -28 & -84 & 28 & 8.28 & 27 & 19 \\
\hline
\end{tabular}

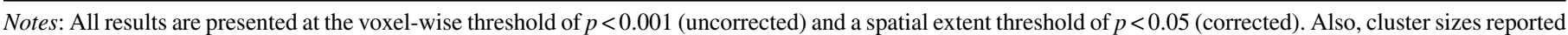
in brackets refer to activations, which were within clusters listed elsewhere in the table.

peak activation ( $x, y$, and $z$ coordinates, see Section 2$)$ across languages were not significant, suggesting that there is no systematic difference in the location of the left prefrontal region being activated in L1 versus L2. The extent of the activation was also not different across languages $(t(7)=0.84, p>0.05)$, suggesting that the size of the LIFG regions activated are the same in L1 and L2.

Tests on the relationship between syntactical proficiency (Toefl scores) and the above measures (strength, location, and extent of the L IFG activations) revealed the following. The correlations between the Toefl scores and the strength of the activations (English: $r=-0.09, p>0.05$, and French: $r=0.16$, $p>0.05)$, the location of the peak activations, and the size of the LIFG clusters (English: $r=0.57, p>0.05$, French $r=0.43$, $p>0.05$ ) were not significant, suggesting that grammatical proficiency in a second language does not predict these measures for LIFG activations in the first nor the second language during the sentence production minus word reading contrast. Finally, we correlated Toefl scores with the distance separating the LIFG peak activation location in English and in French $(N=8)$. Remarkably, there was a significant correlation between the English versus French LIFG peak location distances and the Toefl scores $(r=-0.71, p<0.05)$, indicating that less grammatically proficient bilinguals show a greater separation in the

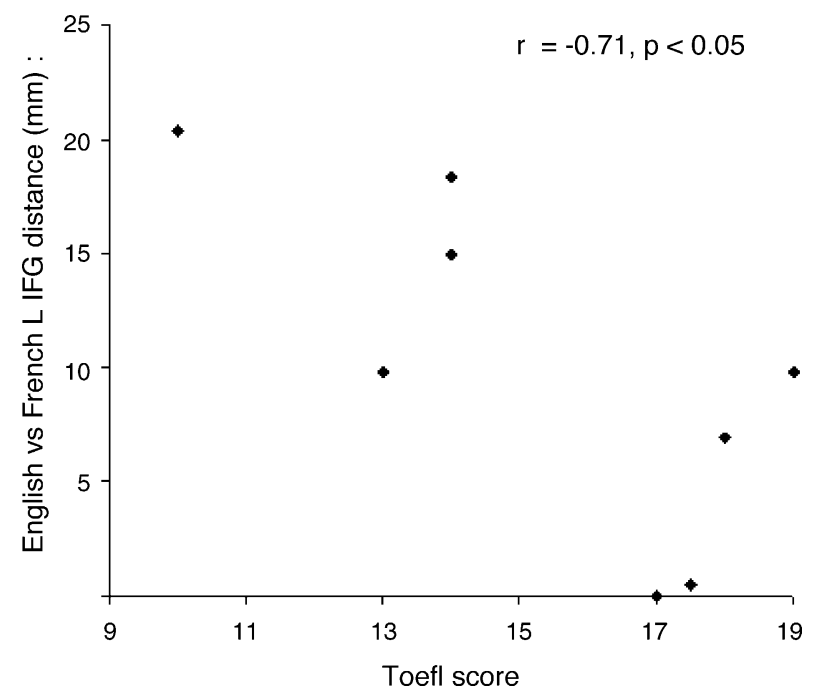

Fig. 3. English vs. French LIFG peak distances vs. proficiency. Scatter plot showing correlation between English vs. French left inferior frontal peak distances and Toefl subtest scores $(r=-0.71, p<0.05)$, showing a greater separation in LIFG peaks in less compared to more proficient bilinguals during the syntactical task.
LIFG peaks across languages during the syntactical task (see Fig. 3).

\section{Discussion}

\subsection{Neuroimaging: group subtractions}

Pre-training behavioural testing during overt production revealed that all subjects were able to perform even the most difficult task involving producing sentences from five words in the second language. The similar pattern of response times obtained across the eight different conditions (two task, two language, and two word length conditions) during overt production, obtained in a behavioural setting, and during covert production, obtained during fMRI scanning, suggests that subjects were indeed performing the task at hand in the latter setting.

Word reading, in both the native and the second language, resulted in activation in regions previously shown to be activated during covert word reading, including the occipital (BA 18) and fusiform (BA 19/37) regions bilaterally. There was also activation in the left putamen in English. This is consistent with findings by Klein et al. (1994), who showed that this region was additionally involved during overt word repetition in L2 compared to L1 in bilingual individuals. It was proposed that this region may be additionally activated due to the increased articulatory demands of producing speech in L2 compared to L1. The fact that we also find activation in this region only in L2 during covert production suggests that activation in this region is related to difficulty even at the planning stage of word production, before overt production is executed.

The production of sentences from a list of words (see Table 2), as well as the sentence production minus word reading 'syntactical' subtraction (see Table 3), also yielded a similar pattern of activation in the first and in the second languages in regions previously implicated in syntax and working memory, in covert language production, and in attention and executive processes. These activations will be discussed in relation to their roles in syntax and working memory, as well as in more general executive, attentional, and motor processes below.

Before we begin, however, we would like to raise a few points. With respect to the sentence production minus word reading subtraction, note that although the comparison between the sentence and the word conditions ought to reveal processes involved in sentence building and syntactical processing, syntactical processing is not isolated in this comparison, and other processes such as semantic evaluation, lexical selection, and phonological 
assembly may also be revealed. Note in addition the inherent difficulty in precisely isolating component language processes in functional imaging experiments, in particular when aiming to isolate syntax during a production task; the production of grammatical sentences must by definition include other levels of language (semantics, phonology, prosody), since the production of syntax independent of these other levels (e.g., production of Jabberwocky sentences) would be highly unnatural and effortful, more so during production than during perception, making the selection of an appropriate control condition particularly challenging if not impossible. Further, given that the sentence generation task requires participants to create sentences from words appearing on the screen, it is likely that this task relies more on working memory resources than word reading. On the other hand, two aspects of the experimental design helped to ensure that working memory requirements for the experimental task were minimized. First, the words remained on the screen throughout each trial. Second, the words were, only in the sentence generation task, presented in the correct order for generating a sentence.

\subsubsection{Syntax and verbal working memory systems}

As can be seen in Tables 2 and 3, Broca's area (BA44) was activated in both languages during the production of sentences, and this activation extended to BA 45 and to the middle frontal gyrus (BA 46) during sentence production in English and during the sentence minus word subtraction in both languages. As described in the introduction, many studies have shown activation in regions in and around Broca's area during syntax processing (Embick et al., 2000; Ni et al., 2000; Wartenburger et al., 2003). Such activation has also been observed in studies examining the functional substrates of syntax processing when employing production tasks (Dogil et al., 2002; Heim et al., 2002; Indefrey et al., 2001; Indefrey et al., 2004). Given evidence for the involvement of this region in verbal working memory (Paulesu, Frith, \& Frackowiak, 1993; Smith, Jonides, Marshuetz, \& Koeppe, 1998), some have suggested that Broca's area is activated during syntax processing when the task at hand places demands on the working memory system (Stromswold, Caplan, Alpert, \& Rauch, 1996).

The left inferior parietal cortex has been implicated in semantic and phonetic levels of language processing in general (Mesulam, 1990), and syntactic comprehension in particular (Caplan, Hildebrandt, \& Makris, 1996; Dapretto \& Bookheimer, 1999). Specifically, it has been shown that the left inferior parietal lobe and the contiguous left posterior superior temporal lobe are activated by complex syntactic structures in experiments that impose a high 'extrinsic' memory load, above and beyond the 'syntactic' memory load associated with processing more complex syntactic structures (Caplan, 2001; Stowe et al., 1998). More specifically, the left supramarginal gyrus has been shown to play a role in the coding and retrieval of order information in verbal working memory (Marshuetz, Smith, Jonides, DeGutis, \& Chenevert, 2000). Broca's area has been posited to perform similar functions during syntactical processing: verbal and maybe more general working memory, and generation of serial-order representations (Keller et al., 2001), and gen- eral sequencing (Rizzolatti \& Arbib, 1998). Taken together, our findings of activation in the left inferior parietal region only in L2 during sentence production and during the sentence production minus word reading subtraction, and concurrent greater activation in the left prefrontal cortex in L2 compared to L1 during the sentence production minus word reading subtraction (see below), suggest that the working memory/sequencing demands are greater in the second compared to the first language of bilinguals. The former finding is interesting in the light of a recent voxel-based morphometry study showing anatomical differences between monolingual and bilingual individuals in the left inferior parietal cortex (Mechelli et al., 2004).

We find activation of the left putamen in the sentence production minus word reading subtraction in the first language. At first glance, this may appear contradictory with the above reported left putamen activation during word reading in $\mathrm{L} 2$. The differential pattern of activation of this structure across languages in the two tasks, however, might be due to different levels of speech. Specifically, during word reading, activation of this region may reflect articulatory demands of word production in L2, whereas in the sentence production minus word reading subtraction, it might reflect syntactical processing in L1. There is functional imaging and electrophysiological evidence for basal ganglia involvement in syntactic processing (Friederici \& Kotz, 2003; Kotz, Frisch, von Cramon, \& Friederici, 2003). Also, lesion studies show the importance of intact subcortical fibers connecting to and from Broca's area for language production more generally. Basal ganglia damage does not, however, always result in language production deficits, suggesting that the specific location and extent of the subcortical lesion determines whether or not language production is affected. Our finding of activation of the left putamen only in the first language during the sentence production minus word reading contrast in bilinguals is consistent with a model of language processing whereby grammatical processes are posited to rely more on a procedural, rule-based memory system than are semantic processes, and are therefore thought to depend more on subcortical structures (Ullman, 2004). This model is extended to bilingualism, where it is proposed that grammatical processing relies more on the procedural memory system in L1 compared to L2, and is therefore more dependent on regions including the basal ganglia (Ullman, 2001).

\subsubsection{Executive and attention systems}

A few studies on syntax have shown activation in regions of the dorsolateral prefrontal cortex (DLPFC) (BA 46, 47, 6, and 9) (Caplan, Waters, \& Alpert, 2003; Wartenburger et al., 2003). Due to variability in the cytoarchitectonic locations of BAs 9 and 46, the BA 46 group activations that we observe may actually extend to or encompass BA 9 (Rajkowska \& Goldman-Rakic, 1995). The DLPFC is thought of as being a component of a supervisory attention and central executive system (Petrides, Alivisatos, Meyer, \& Evans, 1993; Shallice, 1988), and it has been suggested that this region is at the boundary between 'executive' and 'language' regions (Caplan et al., 2003). It has been shown to be activated during intrinsic word generation (Frith, Friston, Liddle, \& Frackowiak, 1991). Intrinsic word generation may 
characterize an aspect of our sentence generation task in that during sentence production, some non-content words had to be added to words presented on the screen for successful task performance. We find greater signal change in regions including BA 46 in the second compared to the first language during the sentence minus word subtraction (see below), suggesting that the executive components of syntactical production are more demanding in L2 compared to L1.

The right cerebellum was activated in both languages during sentence production and only in L2 during the sentence versus word subtraction. There is evidence for cerebellar involvement in language processing (Ackermann \& Hertrich, 2000). More specifically, there is some evidence that lesions to the cerebellum can result in syntax production deficits (Silveri, Leggio, \& Molinari, 1994; Zettin et al., 1997), although similarly to the above reported evidence for BG involvement in language production, damage to the cerebellum does not always result in syntax production deficits, suggesting that the specific nature and extent of damage (e.g., damage to fibres connecting the cerebellum to components of the language network) is critical in determining whether or not specific deficits are observed. There is also some functional imaging evidence for involvement of the cerebellum in syntax processing specifically (Dogil et al., 2002). In domains not specific to language, lesion work suggests that the cerebellum contributes to aspects of executive function and attention including sequential reasoning, which are associated with the functional role of the prefrontal cortex (see Marien, Engelborghs, Fabbro, \& De Deyn (2001) for a review). Interestingly, in relation to the DLPFC activation noted above, there is evidence for anatomical connectivity between the right cerebellum and contralateral prefrontal areas (BA 46) in primates (Middelton \& Strick, 1994). In humans, there is functional imaging evidence for coactivation of the left prefrontal cortex and the contralateral cerebellum during verbal association/word generation tasks (Petersen, Fox, Posner, Mintun, \& Raichle, 1988; Raichle et al., 1994). We find right cerebellar activation in the sentence production versus word reading subtraction in the second but not in the first language. This finding, in conjunction with greater activation of regions including the DLPFC (see below) in L2 compared to L1, suggests differential involvement of processes modulating syntactical processing and its execution in the second versus the first language of moderately fluent bilinguals.

The right posterior intraparietal sulcus (IPS) is activated in both languages and the left IPS in English during sentence production, and the same pattern holds for the sentence production minus word reading subtraction (see Tables 2 and 3). Previous work has shown the involvement of the right intraparietal sulcus in a variety of tasks with high attentional demands (Culham \& Kanwisher, 2001). Attentional orienting (Corbetta \& Shulman, 2002) is thought to be dependent on bilateral activity in the intraparietal sulcus. The superior parietal lobe has also been shown to be involved in switching between language tasks (Gurd et al., 2002). Activation in the left IPS only in English during both the sentence production and in the sentence production minus word reading subtraction suggests that the attentional and/or 'executive' aspects of sentence production are more demanding in the second compared to the first language.

\subsubsection{Effect of number of words}

In the word reading condition, there was a significant effect of number of words in the left occipital and fusiform regions, but not in the LIFG. This observation is relevant to the discussion of a potential confound in our study: as already described, subjects produced more words in the 'sentence' compared to the 'word' conditions, since they added non-content words when necessary during the former but not during the latter condition. Consistent with previous findings (Caplan et al., 2000), the absence of an effect of number of words in Broca's area during the wordreading task suggests that activation in this region during the production of grammatical sentences is not due to the somewhat different articulatory demands across tasks.

\subsection{Functional correlates of proficiency}

We find that syntactical proficiency in English, as measured by the Structure subtest of the Toefl which measures grammatical proficiency, is positively correlated with the degree to which the left caudate nucleus/putamen is recruited across subjects during both the sentence production minus word reading subtraction and during sentence production. Scatter plots showing the signal change in this region during the sentence production minus word reading subtraction suggest that less proficient subjects deactivate the basal ganglia more than more proficient ones (not shown). Similar plots looking at the main effect of sentences (not shown) suggest that more proficient subjects recruit the basal ganglia and that less proficient subjects suppress this region. Within the framework of Ullman's model described above (Ullman, 2004), this could suggest that bilinguals who are more grammatically proficient use a more 'rule-based', procedural, native-like language processing system than the less syntactically proficient bilinguals during the production of sentences in a second language, in other words, during language production generally, whether at the semantic, syntactic, phonetic, or prosodic levels.

\subsection{Differential involvement of broca's area in L1 versus L2}

In the group subtractions, we did not find activation in the LIFG in the English minus French, sentences minus words subtraction (i.e., in the task by language interaction), maybe due to inter-subject variability in the location of the left prefrontal activation. The opposite interaction, French minus English, sentences minus words, yielded activation in the orbitofrontal cortex. This was not expected, and if anything, the opposite might have been predicted (i.e., more activation in this region in English compared to French), since the task is likely more difficult in L2 compared to in L1. From analysis of negative BOLD results (not shown), however, it appears that this result could be due to greater deactivation in this region during English compared to French sentence production. The orbitofrontal cortex is part of the resting baseline network, and it is possible that our subjects deactivate this region more in English than in French because they allocate more neural resources to task relevant modalities and functions. 
Analyses on the signal change in left frontal activation clusters identified on a subject-by-subject basis revealed greater activation in L2 compared to L1 during sentence production and during word reading. There was no task by language interaction, suggesting that greater activation in L2 compared to L1 may occur at any level of language during language generation tasks. This result fits with several lines of work showing that (a) more novel, effortful, 'top-down' task performance recruits frontal regions more than when the task is more practiced, easier, and bottom-up (Frith et al., 1991; Raichle et al., 1994), and (b) more specifically, that during the performance of semantic tasks (Chee et al., 2001) and of phonetic learning tasks (Golestani \& Zatorre, 2004), lesser proficiency or poorer task performance or learning is associated with greater recruitment of frontal regions. Similarly to as has been proposed for semantic processing (Chee et al., 2001), it is possible that lesser proficiency in L2 is associated with greater cognitive effort, which may be subserved by less well-tuned neural representations that require greater neuronal activity than during processing in L1. Alternatively and not exclusively, greater LIFG activation in L2 compared to L1 could be related to differences in word retrieval effort between the native and second languages. It has been shown that low frequency words result in greater left prefrontal BOLD signal change than high frequency words (Chee, Hon, Caplan, Lee, \& Goh, 2002). It can be expected that due to greater familiarity with the native language, experience-dependent frequency rank order of words in L2 is lower than in L1. It is therefore possible that greater retrieval effort for words in L2 compared to L1 in part drives the differential degree of functional involvement of the left prefrontal region.

As mentioned in the introduction, to our knowledge, only one other study has been done examining the neural correlates of syntax in bilinguals (Wartenburger et al., 2003). In contrast to our study, this study was done using a receptive task, and involved detection of syntactic or of semantic anomalies. It was found that during the grammatical but not during the semantic task, late proficient bilinguals activate left inferior frontal regions bilaterally more than do early proficient bilinguals. In other words, they found an effect of age of acquisition on left prefrontal activation. We have found a similar result between languages and within subjects during sentence production more generally; there is more activation in the left prefrontal cortex in the language learned after the age of 10 compared to in the native language. Wartenburger et al. (2003) did not find an effect of proficiency on activation in the left prefrontal cortex during the syntactical task. Based both on correlations between the BOLD signal in the syntactical contrast with Toefl scores, and on the correlation of the signal in the individually defined left prefrontal ROI and Toefl scores, neither did we. Both of these negative findings might be because individual differences in proficiency within the same language may not have been large enough to result in detectable functional differences. In Wartenburger et al. study, it is also possible that individual differences in the location of the left prefrontal activations were too important to allow and effect of proficiency to be detected in a group comparison. We, however, did not find an effect of proficiency on LIFG activations even when taking such individual differences into account.
Alternatively, it is possible that proficiency does not modulate the strength of left prefrontal activation during syntactical tasks. Given the above-mentioned work showing greater left prefrontal activation in the less proficient language and during poorer task performance or learning during semantic and phonological tasks (Golestani \& Zatorre, 2004), however, this would suggest that syntax is different from other levels of language with respect to the impact of proficiency or ease of task performance on frontal brain activity.

\subsection{English versus French LIFG distances}

Individual subject analyses on the left frontal region also showed that less grammatically proficient subjects show a greater separation in the peak location of French and English syntactical LIFG activation than do more proficient ones. This finding constitutes an extension of that by Kim et al. (1997), who showed an effect of age of acquisition on the distance separating left frontal activations in the first versus the second language during a covert sentence production task. We show a similar result for proficiency, and our results are the first, to our knowledge, to show that proficiency in a second language can influence the pattern of brain activation during a production task designed to favour syntax processing. A question to be addressed in future research is the mechanisms of such a finding. For example, it is possible that either the acquisition of a second language early in life, and/or the attainment of more 'automatic' skills in a second language due to training and practice is accompanied by a shift or 'convergence' of left prefrontal activations to regions that are more 'specialised' or 'expert' for aspects of language processing, and which are ones recruited during the processing of the native language. Another question to be addressed is whether such a finding may apply to levels of language other than syntax. Due to work showing that syntax and phonetics are the levels of language most vulnerable to delays in exposure (i.e., ones more likely involving a 'critical period') (Rivera-Gaxiola, Silva-Pereyra, \& Kuhl, 2005; Weber-Fox \& Neville, 1996), it is possible that early acquisition of and/or greater skill in the processing of speech sounds belonging to a second language is also accompanied by such a 'shift' towards to the recruitment of posited more 'expert' prefrontal regions, ones recruited during the processing of native speech sounds.

\subsection{Summary and conclusions}

Functional imaging during covert word reading showed the involvement of regions previously shown to be activated during covert reading, such as the occipital (BA 18) and fusiform (BA 19/37) regions bilaterally. Syntactical production activated regions previously implicated in syntax, working memory, and executive and attentional processes including Broca's area (BA 44/45), the dorsolateral prefrontal cortex, and the right superior parietal cortex in both the first and second language in moderately fluent bilinguals. In the first language, there was additional activation in the left putamen, and in the second language, there was additional activation of the left inferior and superior parietal cortices, and of the right occipital cortex and cerebellum. There 
was greater left prefrontal activation in the second compared to the first language, suggesting that processing in these regions is more efficient and/or may require less processing time in the first compared to the second language during the production of grammatical sentences.

Our participants differed in their level of grammatical proficiency in the second language. We found that more proficient bilinguals engage a component of the procedural memory system more than do less proficient individuals, suggesting that they engage in more rule-based, native-like processing during sentence production in a second language. Most importantly, we found that during syntactical production, left prefrontal activation peaks are closer in L1 and L2 in more compared to less grammatically proficient bilinguals. This result could be due to individual differences in the 'ability' to engage cortex that is optimized for aspects of syntactical processing during syntax production in the second language. In other words, maybe more grammatically proficient bilinguals use cortex that is more 'tuned' for native-like processing, due for example to its architectonics and pattern of connectivity, even during the processing of a language learned later in life, after a critical period. Alternatively, it is possible that individuals differ in the critical period window for aspects of second language learning, ones that result in differences in the degree to which individuals recruit more 'optimized' neural representations or processes, even during processing of a second language. Future work will be needed to address these issues.

\section{Acknowledgements}

We acknowledge the support of the French Ministry of Research through an ACI grant. This work has been presented at CNS 2005.

\section{References}

Ackermann, H., \& Hertrich, I. (2000). The contribution of the cerebellum to speech processing. Journal of Neurolinguistics, 13(2/3), 95-116.

Birdsong, D. (1999). Second language acquisition and the critical period hypothesis. New Jersey: Lawrence Erlbaum Associates.

Bookheimer, S., Zeffiro, T. A., Blaxton, T., Gaillard, W., \& Theodore, W. (1995). Regional cerebral blood flow during object naming and word reading. Human Brain Mapping, 3(2), 93-106.

Buckner, R. L., Koutstaal, W., Schacter, D. L., \& Rosen, B. R. (2000). Functional MRI evidence for a role of frontal and inferior temporal cortex in amodal components of priming. Brain, 123(Pt 3), 620-640.

Caplan, D. (2001). Functional neuroimaging studies of syntactic processing. Journal of Psycholinguistic Research, 30(3), 297-320.

Caplan, D., Alpert, N., \& Waters, G. (1998). Effects of syntactic structure and propositional number on patterns of regional cerebral blood flow. Journal of Cognitive Neuroscience, 10(4), 541-552.

Caplan, D., Alpert, N., Waters, G., \& Olivieri, A. (2000). Activation of Broca's area by syntactic processing under conditions of concurrent articulation. Human Brain Mapping, 9(2), 65-71.

Caplan, D., Hildebrandt, N., \& Makris, N. (1996). Location of lesions in stroke patients with deficits in syntactic processing in sentence comprehension. Brain, 119(Pt 3), 933-949.

Caplan, D., Waters, G., \& Alpert, N. (2003). Effects of age and speed of processing on $\mathrm{rCBF}$ correlates of syntactic processing in sentence comprehension. Human Brain Mapping, 19(2), 112-131.
Chee, M. W., Caplan, D., Soon, C. S., Sriram, N., Tan, E. W., Thiel, T., et al. (1999). Processing of visually presented sentences in Mandarin and English studied with fMRI. Neuron, 23(1), 127-137.

Chee, M. W., Hon, N., Lee, H. L., \& Soon, C. S. (2001). Relative language proficiency modulates BOLD signal change when bilinguals perform semantic judgments. Blood oxygen level dependent. Neuroimage, $13(6$ Pt 1), 1155-1163.

Chee, M. W., Hon, N. H., Caplan, D., Lee, H. L., \& Goh, J. (2002). Frequency of concrete words modulates prefrontal activation during semantic judgments. Neuroimage, 16(1), 259-268.

Cooke, A., Zurif, E. B., DeVita, C., Alsop, D., Koenig, P., Detre, J., et al. (2002). Neural basis for sentence comprehension: Grammatical and short-term memory components. Human Brain Mapping, 15(2), 80-94.

Corbetta, M., \& Shulman, G. L. (2002). Control of goal-directed and stimulus-driven attention in the brain. Nature Reviews in the Neurosciences, 3(3), 201-215.

Culham, J. C., \& Kanwisher, N. G. (2001). Neuroimaging of cognitive functions in human parietal cortex. Current Opinion in Neurobiology, 11(2), 157-163.

Dapretto, M., \& Bookheimer, S. Y. (1999). Form and content: Dissociating syntax and semantics in sentence comprehension. Neuron, 24(2), 427-432.

Dehaene, S., Dupoux, E., Mehler, J., Cohen, L., Paulesu, E., Perani, D., et al. (1997). Anatomical variability in the cortical representation of first and second language. Neuroreport, 8(17), 3809-3815.

Dogil, G., Ackermann, H., Grodd, W., Haider, H., Kamp, H., Mayer, J., et al. (2002). The speaking brain: A tutorial introduction to fMRI experiments in the production of speech, prosody and syntax. Journal of Neurolinguistics, 15(1), 59-90.

Embick, D., Marantz, A., Miyashita, Y., O'Neil, W., \& Sakai, K. L. (2000). A syntactic specialization for Broca's area. Proceedings of the National Academy of Sciences of the United States of America, 97(11), 6150-6154.

Fiez, J. A. (1997). Phonology, semantics, and the role of the left inferior prefrontal cortex. Human Brain Mapping, 5(2), 79-83.

Friederici, A. D. (2004). Processing local transitions versus long-distance syntactic hierarchies. Trends in Cognitive Sciences, 8(6), 245-247.

Friederici, A. D., \& Kotz, S. A. (2003). The brain basis of syntactic processes: Functional imaging and lesion studies. Neuroimage, 20(Suppl. 1), S8-S17.

Friston, K., Holmes, A. P., Worsley, K. J., Poline, J. B., Frith, C. D., \& Frackowiak, R. S. J. (1995). Statistical parametric maps in functional imaging: A general linear approach. Human Brain Mapping, 2, 189-210.

Frith, C. D., Friston, K. J., Liddle, P. F., \& Frackowiak, R. S. (1991). A PET study of word finding. Neuropsychologia, 29(12), 1137-1148.

Golestani, N., \& Zatorre, R. J. (2004). Learning new sounds of speech: Reallocation of neural substrates. Neuroimage, 21(2), 494-506.

Gurd, J. M., Amunts, K., Weiss, P. H., Zafiris, O., Zilles, K., Marshall, J. C., et al. (2002). Posterior parietal cortex is implicated in continuous switching between verbal fluency tasks: An fMRI study with clinical implications. Brain, 125(Pt 5), 1024-1038.

Heim, S., Opitz, B., \& Friederici, A. D. (2002). Broca's area in the human brain is involved in the selection of grammatical gender for language production: Evidence from event-related functional magnetic resonance imaging. Neuroscience Letters, 328(2), 101-104.

Illes, J., Francis, W. S., Desmond, J. E., Gabrieli, J. D., Glover, G. H., Poldrack, R., et al. (1999). Convergent cortical representation of semantic processing in bilinguals. Brain and Language, 70(3), 347-363.

Indefrey, P., Brown, C. M., Hellwig, F., Amunts, K., Herzog, H., Seitz, R. J., et al. (2001). A neural correlate of syntactic encoding during speech production. Proceedings of the National Academy of Sciences of the United States of America, 98(10), 5933-5936.

Indefrey, P., Hellwig, F., Herzog, H., Seitz, R. J., \& Hagoort, P. (2004). Neural responses to the production and comprehension of syntax in identical utterances. Brain and Language, 89(2), 312-319.

Joubert, S., Beauregard, M., Walter, N., Bourgouin, P., Beaudoin, G., Leroux, J. M., et al. (2004). Neural correlates of lexical and sublexical processes in reading. Brain and Language, 89(1), 9-20.

Jusczyk, P. W. (1995). Language acquisition: Speech sounds and the beginning of phonology. In J. L. Miller \& P. D. Eimas (Eds.), Speech, language, and communication. Academic Press. 
Kaan, E., \& Swaab, T. Y. (2002). The brain circuitry of syntactic comprehension. Trends in Cognitive Sciences, 6(8), 350-356.

Keller, T. A., Carpenter, P. A., \& Just, M. A. (2001). The neural bases of sentence comprehension: A fMRI examination of syntactic and lexical processing. Cerebral Cortex, 11(3), 223-237.

Kherif, F., Poline, J. B., Meriaux, S., Benali, H., Flandin, G., \& Brett, M. (2003). Group analysis in functional neuroimaging: Selecting subjects using similarity measures. Neuroimage, 20(4), 2197-2208.

Kim, K. H., Relkin, N. R., Lee, K. M., \& Hirsch, J. (1997). Distinct cortical areas associated with native and second languages. Nature, 388(6638), 171-174.

Klein, D., Zatorre, R. J., Milner, B., Meyer, E., \& Evans, A. C. (1994). Left putaminal activation when speaking a second language: Evidence from PET. Neuroreport, 5(17), 2295-2297.

Klingberg, T., Kawashima, R., \& Roland, P. E. (1965-1971). Activation of multi-modal cortical areas underlies short-term memory. European Journal of Neuroscience, 8(9).

Kotz, S. A., Frisch, S., von Cramon, D. Y., \& Friederici, A. D. (2003). Syntactic language processing: ERP lesion data on the role of the basal ganglia. Journal of International Neuropsychological Society, 9(7), 1053-1060.

Kuhl, P. K. (2000). A new view of language acquisition. Proceedings of the National Academy of Sciences of the United States of America, 97(22), 11850-11857.

Marien, P., Engelborghs, S., Fabbro, F., \& De Deyn, P. P. (2001). The lateralized linguistic cerebellum: A review and a new hypothesis. Brain and Language, 79(3), 580-600.

Marshuetz, C., Smith, E. E., Jonides, J., DeGutis, J., \& Chenevert, T. L. (2000). Order information in working memory: fMRI evidence for parietal and prefrontal mechanisms. Journal of Cognitive Neuroscience, 12(Suppl. 2), 130-144.

Mechelli, A., Crinion, J. T., Noppeney, U., O’Doherty, J., Ashburner, J., Frackowiak, R. S., et al. (2004). Neurolinguistics: Structural plasticity in the bilingual brain. Nature, 431(7010), 757.

Mesulam, M. M. (1990). Large-scale neurocognitive networks and distributed processing for attention, language, and memory. Annals of Neurology, 28(5), 597-613.

Middelton, F., \& Strick, P. (1994). Anatomical evidence for cerebellar and basal ganglia involvement in higher cognitive functions. Science, 266, 458-461.

Müller, R. A., \& Basho, S. (2004). Are non-linguistic functions in "Broca's area" prerequisites for language acquisition? fMRI findings from an ontogenetic viewpoint. Brain and Language, 89, 329-336.

Ni, W., Constable, R. T., Mencl, W. E., Pugh, K. R., Fulbright, R. K., Shaywitz, S. E., et al. (2000). An event-related neuroimaging study distinguishing form and content in sentence processing. Journal of Cognitive Neuroscience, 12(1), 120-133.

Paulesu, E., Frith, C. D., \& Frackowiak, R. S. (1993). The neural correlates of the verbal component of working memory. Nature, 362(6418), 342-345.

Perani, D., Paulesu, E., Galles, N. S., Dupoux, E., Dehaene, S., Bettinardi, V., et al. (1998). The bilingual brain. Proficiency and age of acquisition of the second language. Brain, 121(Pt 10), 1841-1852.

Petersen, S. E., Fox, P. T., Posner, M. I., Mintun, M., \& Raichle, M. E. (1988). Positron emission tomographic studies of the cortical anatomy of single-word processing. Nature, 331(6157), 585-589.
Petrides, M., Alivisatos, B., Meyer, E., \& Evans, A. C. (1993). Functional activation of the human frontal cortex during the performance of verbal working memory tasks. Proceedings of the National Academy of Sciences of the United States of America, 90(3), 878-882.

Raichle, M. E., Fiez, J. A., Videen, T. O., MacLeod, A. M., Pardo, J. V., Fox, P. T., et al. (1994). Practice-related changes in human brain functional anatomy during non-motor learning. Cerebral Cortex, 4(1), 8-26.

Rajkowska, G., \& Goldman-Rakic, P. S. (1995). Cytoarchitectonic definition of prefrontal areas in the normal human cortex: II. Variability in locations of areas 9 and 46 and relationship to the Talairach Coordinate System. Cerebral Cortex, 5(4), 323-337.

Rivera-Gaxiola, M., Silva-Pereyra, J., \& Kuhl, P. K. (2005). Brain potentials to native and non-native speech contrasts in 7- and 11-month-old American infants. Developmental Science, 8(2), 162-172.

Rizzolatti, G., \& Arbib, M. A. (1998). Language within our grasp. Trends in Neurosciences, 21(5), 188-194.

Shallice, T. (1988, October 28). From neuropsychology to mental structure. Cambridge, MA: Cambridge University Press.

Silveri, M. C., Leggio, M. G., \& Molinari, M. (1994). The cerebellum contributes to linguistic production: A case of agrammatic speech following a right cerebellar lesion. Neurology, 44(11), 2047-2050.

Smith, E. E., Jonides, J., Marshuetz, C., \& Koeppe, R. A. (1998). Components of verbal working memory: Evidence from neuroimaging. Proceedings of the National Academy of Sciences of the United States of America, 95(3), 876-882.

Stowe, L. A., Broere, C. A., Paans, A. M., Wijers, A. A., Mulder, G., Vaalburg, W., et al. (1998). Localizing components of a complex task: Sentence processing and working memory. Neuroreport, 9(13), 2995-2999.

Stromswold, K., Caplan, D., Alpert, N., \& Rauch, S. (1996). Localization of syntactic comprehension by positron emission tomography. Brain and Language, 52(3), 452-473.

Tettamanti, M., Alkadhi, H., Moro, A., Perani, D., Kollias, S., \& Weniger, D. (2002). Neural correlates for the acquisition of natural language syntax. Neuroimage, 17(2), 700-709.

Ullman, M. T. (2001). The neural basis of lexicon and grammar in first and second language: The declarative/procedural model. Bilingualism: Language and Cognition, 4(1), 105-122.

Ullman, M. T. (2004). Contributions of memory circuits to language: The declarative/procedural model. Cognition, 92(1/2), 231-270.

Vingerhoets, G., Van Borsel, J., Tesink, C., van den Noort, M., Deblaere, K., Seurinck, R., et al. (2003). Multilingualism: An fMRI study. NeuroImage, 20, 2181-2196.

Wagner, A. D., Pare-Blagoev, E. J., Clark, J., \& Poldrack, R. A. (2001). Recovering meaning: Left prefrontal cortex guides controlled semantic retrieval. Neuron, 31(2), 329-338.

Wartenburger, I., Heekeren, H. R., Abutalebi, J., Cappa, S. F., Villringer, A., \& Perani, D. (2003). Early setting of grammatical processing in the bilingual brain. Neuron, 37(1), 159-170.

Weber-Fox, C., \& Neville, H. J. (1996). Maturational constraints on functional specializations for language processing: ERP and behavioral evidence in bilingual speakers. Journal of Cognitive Neuroscience, 8, 231-256.

Zettin, M., Cappa, S. F., D’Amico, A., Rago, R., Perino, C., Perani, D., et al. (1997). Agrammatic speech production after a right cerebellar haemorrhage. Neurocase, 3, 375-380. 